October 19, 2018

\title{
Hopf instantons in Chern-Simons theory
}

\author{
C. Adam* \\ Institut für theoretische Physik, Universität Karlsruhe \\ B. Muratori**, C. Nash*** \\ Department of Mathematical Physics, National University of Ireland, Maynooth
}

\begin{abstract}
We study an Abelian Chern-Simons \& Fermion system in three dimensions. In the presence of a fixed prescribed background magnetic field we find an infinite number of fully three-dimensional solutions. These solutions are related to Hopf maps and are, therefore, labelled by the Hopf index. Further we discuss the interpretation of the background field.
\end{abstract}

*)email address: adam@maths.tcd.ie, adam@pap.univie.ac.at

**)email address: bmurator@fermi1.thphys.may.ie

***)email address: cnash@stokes2.thphys.may.ie 


\section{Introduction}

It is the purpose of this letter to construct an infinite number of fully three-dimensional solutions to a Chern-Simons \& Fermion system that are labelled by the Hopf index, thereby merging two features - Chern-Simons theory and Hopf maps - that have recently received wide attention within quantum field theory.

On the one hand, Hopf maps are just maps $S^{3} \rightarrow S^{2}$. These maps fall into different homotopy classes that are labelled by the integers (the Hopf index, see below for details). Field configurations with nontrivial Hopf index have already been studied for some time in fluid dynamics [1], in astrophysics [2, 3], in magnetic solids (magnetic solitons, see e.g., [4]) and in classical electro-magnetism [5]. Recently, there has been a lot of interest in field theories where static solutions with nontrivial Hopf index (Hopf solitons) occur (see e.g., [7]-[12]).

On the other hand, Chern-Simons theories with matter and/or a Maxwell term have been studied intensively since their introduction [13, 14. When an Abelian Chern-Simons term in three dimensions is coupled to matter, the magnetic field is forced to be proportional to the electric current due to the equations of motion [15]-18. Further, in these models there exist soliton-like, static (i.e., two-dimensional) solutions that are related to some topological invariants (e.g., maps $S^{2} \rightarrow S^{2}$ ) [15]- [18]. Usually, these solitons behave like vortices, and, because of their topological nature, they exhibit magnetic flux quantization. Therefore these solutions are physically relevant in situations where the phenomenon of magnetic flux quantization occurs and where matter is confined to a plane, the most prominent example being the quantum Hall effect [19, 20].

At this point the question arises whether there exist fully three-dimensional solutions for such Chern-Simons \& matter systems, and whether these solutions may be characterized by some topological invariants, as well.

In this letter we shall demonstrate that, if the presence of a fixed, prescribed background magnetic field is assumed, then there indeed exist solutions to the Chern-Simons \& Fermion system defined below. Further, these solutions are related to Hopf maps and are, therefore, labelled by the Hopf index. Hence we find, on one hand, a three-dimensional version of the phenomenon of magnetic flux quantization, namely topologically quantized magnetic knots (Hopf maps are related to magnetic knots, see [5, 6]). On the other hand, a Chern-Simons term is well-known to be induced in $\mathrm{QED}_{3}$ (see e.g. [14, 21, 22, 23, 24]). Further, a Chern-Simons term is used for topological generation of mass in some threedimensional QFTs 13, 14. Whenever Fermions are included in such theories, our solutions (the Hopf instantons) should be relevant for the study of non-perturbative features of these QFTs (as well as of $\mathrm{QED}_{3}$ ).

In the main section of this letter we define the model and construct solutions to its equations of motion, as well as the Hopf maps that provide these solutions. In the final section we give two possible interpretations for the background field that is present in our solutions. 


\section{Construction of the Hopf instantons}

We start with the action $(i, j, k=1 \ldots 3)$

$$
S=\int d^{3} x\left(\Psi^{\dagger}\left(-i \partial_{j}-\bar{A}_{j}\right) \sigma_{j} \Psi+\frac{1}{2} \vec{A} \vec{B}\right)
$$

where $\Psi$ is a two-component spinor (Fermion), $\sigma_{j}$ are the Pauli matrices and $\vec{A}$ is an Abelian gauge potential. Further,

$$
S_{\mathrm{CS}}=\frac{1}{2} \int d^{3} x \vec{A} \vec{B}=\frac{1}{4} \int d^{3} x \epsilon_{i j k} A_{i} F_{j k}
$$

is the Chern-Simons (CS) action, where the Chern-Simons coupling constant is chosen equal to one; and

$$
\bar{A}_{i}=A_{i}+A_{i}^{\mathrm{B}}
$$

where the background gauge field $A_{i}^{\mathrm{B}}$ and its magnetic field $B_{i}^{\mathrm{B}}=\epsilon_{i j k} \partial_{j} A_{k}^{\mathrm{B}}$ are

$$
\vec{A}^{\mathrm{B}}=-\frac{1}{1+r^{2}} \vec{N}, \quad \vec{B}^{\mathrm{B}}=-\frac{4}{\left(1+r^{2}\right)^{2}} \vec{N}
$$

and we have introduced the unit vector

$$
\vec{N}=\frac{1}{1+r^{2}}\left(\begin{array}{c}
2 x_{1} x_{3}-2 x_{2} \\
2 x_{2} x_{3}+2 x_{1} \\
1-x_{1}^{2}-x_{2}^{2}+x_{3}^{2}
\end{array}\right)
$$

$\left(\vec{N}^{2}=1\right)$ for later convenience. Observe that the (fixed, non-dynamical) background field is coupled to the Fermion, but it is absent in the CS term. The equations of motion resulting from the action (1) are

$$
\left(-i \partial_{j}-\bar{A}_{j}\right) \sigma_{j} \Psi=0
$$

(the Dirac equation) and

$$
\vec{\Sigma}:=\Psi^{\dagger} \vec{\sigma} \Psi=\vec{B}
$$

Observe that for any pair $\left(\Psi, \bar{A}_{j}\right)$ that solves the Dirac equation (6) the spin density $\vec{\Sigma}$ related to $\Psi$ has to obey

$$
\vec{\partial} \vec{\Sigma}=0
$$

therefore, equations (6) and (7) are consistent.

The simplest solution to this system is (see [25, 26])

$$
\begin{gathered}
\Psi=\frac{4}{\left(1+r^{2}\right)^{\frac{3}{2}}}(\mathbf{1}+i \vec{x} \vec{\sigma})\left(\begin{array}{l}
1 \\
0
\end{array}\right) \\
\vec{A}=\frac{4}{1+r^{2}} \vec{N}
\end{gathered}
$$




$$
\vec{\Sigma}=\vec{B}=\frac{16}{\left(1+r^{2}\right)^{2}} \vec{N}
$$

( $\vec{N}$ is given in (5)). Here the dynamical gauge field is proportional to the background field, therefore one could find a solution without background field by choosing either a different normalization of the fermion (9) or by choosing a Chern-Simons coupling constant in (2), (7) different from 1. However, this will not be true for the solutions below, for which the background field (4) is crucial.

Concerning the geometrical behaviour of the magnetic field $\vec{B}$ in (11), it is important to note that it is related to a Hopf map, and, therefore, the value of the CS action (2) is topologically quantized by the Hopf index. This we want to explain now.

Generally, a complex function $\chi: \mathbf{R}^{3} \rightarrow \mathbf{C}$ with the additional property $\lim _{r \rightarrow \infty} \chi=$ $\chi_{0}=$ const. defines a Hopf map $\chi: S^{3} \rightarrow S^{2}$, where the coordinates in $\mathbf{R}^{3}$ and $\mathbf{C}$ are stereographic coordinates of the $S^{3}$ and $S^{2}$, respectively. The pre-images in $\mathbf{R}^{3}$ of points $\chi=$ const. are closed curves in $\mathbf{R}^{3}$ (circles in $S^{3}$ ), and any two different circles are linked exactly $N$ times, where $N$ is the Hopf index. Further, a magnetic field $\vec{B}$ (the Hopf curvature) is related to the Hopf map $\chi$ via

$$
\vec{B}=\frac{2}{i} \frac{(\vec{\partial} \bar{\chi}) \times(\vec{\partial} \chi)}{(1+\bar{\chi} \chi)^{2}}=4 \frac{S(\vec{\partial} S) \times \vec{\partial} \sigma}{\left(1+S^{2}\right)^{2}}
$$

where $\chi=S e^{i \sigma}$ is expressed in terms of its modulus $S$ and phase $\sigma$ at the r.h.s. of (12).

Mathematically, the curvature $F=\frac{1}{2} F_{i j} d x_{i} d x_{j}, F_{i j}=\epsilon_{i j k} B_{k}$, is the pullback under the Hopf map, $F=\chi^{*} \Omega$, of the standard area two-form $\Omega$ on the target $S^{2}$ with radius 1 (in stereographic coordinates) [5, 0, 10],

$$
\Omega \equiv \frac{2}{i} \frac{d \bar{z} d z}{(1+z \bar{z})^{2}}
$$

Geometrically, $\vec{B}$ is tangent to the closed curves $\chi=$ const. The Hopf index $N$ of $\chi$ may be computed from $\vec{B}$ via

$$
N=\frac{1}{16 \pi^{2}} \int d^{3} x \vec{A} \vec{B}
$$

where $\vec{B}=\vec{\partial} \times \vec{A}$.

The simplest (standard) Hopf map $\chi^{(1)}$ with Hopf index $N=1$ is

$$
\chi^{(1)}=\frac{2\left(x_{1}+i x_{2}\right)}{2 x_{3}-i\left(1-r^{2}\right)}
$$

(a Hopf map has to be single valued, but may well be singular, as $\chi=\infty$ is just the south pole of the target $S^{2}$ ). This $\chi^{(1)},(15)$, leads to a Hopf curvature $\vec{B}^{(1)}$ via (12) that is just the magnetic field (11). Here the question arises whether there are more solutions to (6), (7) that are characterized by higher Hopf maps, and we will find that this is indeed the case. 
[Remark: there is some arbitrariness in the choice of the normalization factor in front of the area two-form (13). We chose it for a target two-sphere of radius one. Consequently, the magnetic field (11) is the Hopf curvature of the standard Hopf map (15) with Hopf index 1, and the background field (4) is minus 1/4 the Hopf curvature of the standard map (15). There are other choices in the literature, e.g. for target two-spheres with radius $1 / \sqrt{2}$ or $1 / 2$. E.g., for radius $1 / \sqrt{2}$ the magnetic field (11) is twice the Hopf curvature of the standard Hopf map (15) (and the background field (4) is minus 1/2 the Hopf curvature of the standard map (15)). Such higher (integer) multiples of $\vec{B}^{(1)}$ may be expressed by higher Hopf maps as follows. Rewrite $\chi^{(1)}$ as $\chi^{(1)}=S \exp (i \sigma)$, where $S$ and $\sigma$ are the modulus and phase of $\chi^{(1)}$ respectively. Then $n \vec{B}^{(1)}$ may be computed via (12) from $\chi_{n}^{(1)}=S \exp (i n \sigma)$. Here only integer $n$ are allowed, because for a Hopf map with nonzero Hopf index the phase $\sigma$ necessarily is multiply valued (see [5] for details). It follows that the background field (4) is not a Hopf curvature (but a fraction thereof) in our choice of conventions.]

Next we should provide some more Hopf maps that will give rise to more solutions to (6), (7). We will produce these Hopf maps by composing the standard Hopf map with some maps $S^{2} \rightarrow S^{2}$, i.e.,

$$
\chi_{R}: S^{3} \stackrel{\chi^{(1)}}{\rightarrow} S^{2} \stackrel{R}{\rightarrow} S^{2} .
$$

In fact, as we use stereographic coordinates on $S^{2}$, we will use rational maps for $R$,

$$
R: z \rightarrow R(z)=\frac{P(z)}{Q(z)}
$$

which are well-known to produce maps $S^{2} \rightarrow S^{2}$ (here $P, Q$ are polynomials) 27, 28]. The winding number of the map is equal to the degree of the rational map, $\operatorname{deg}(R)$,

$$
\operatorname{deg}(R)=\max (p, q)
$$

where $p$ and $q$ are the degrees of the polynomials $P$ and $Q$. In fact, we want to restrict to the simplest maps

$$
R_{n}(z)=z^{n}
$$

i.e., we map

$$
\chi^{(n)}=\left(\chi^{(1)}\right)^{n}
$$

or, for modulus $S=: T^{1 / 2}$ and phase $\sigma$ of $\chi^{(1)}$,

$$
(S, \sigma) \rightarrow\left(S^{n}, n \sigma\right)
$$

where the modulus and phase of the standard Hopf map (15) are

$$
\begin{gathered}
T:=S^{2}=\frac{4\left(r^{2}-x_{3}^{2}\right)}{4 x_{3}^{2}+\left(1-r^{2}\right)^{2}} \\
\sigma=\sigma^{(1)}+\sigma^{(2)} \quad \sigma^{(1)}=\arctan \frac{x_{2}}{x_{1}} \sigma^{(2)}=\arctan \frac{1-r^{2}}{2 x_{3}} .
\end{gathered}
$$


Hopf maps that are composed like (16) lead to a Hopf index $N=n^{2}$, where $n$ is the winding number (i.e., degree) of the rational map (see e.g. [6], where these Hopf maps have been discussed).

For the Hopf curvature this implies

$$
B_{i}^{(n)}=2 \epsilon_{i j k} \frac{\left(T^{n}\right)_{, j} n \sigma_{, k}}{\left(1+T^{n}\right)^{2}}=n^{2} \frac{T^{n-1}(1+T)^{2}}{\left(1+T^{n}\right)^{2}} B_{i}^{(1)},
$$

where $B_{i}^{(1)}$ is just the standard Hopf curvature (11). The logarithm of the factor in front of $B_{i}^{(1)}$ at the r.h.s. of (23), when viewed as a function of $T$,

$$
M_{n}(T) \equiv \ln n^{2} \frac{T^{n-1}(1+T)^{2}}{\left(1+T^{n}\right)^{2}}
$$

obeys the following nonlinear differential equation $\left({ }^{\prime} \equiv \partial / \partial T\right)$

$$
M^{\prime}+T M^{\prime \prime}=-2 \frac{e^{M}-1}{(1+T)^{2}}
$$

for all $n$, as may be checked easily.

Next we need some facts about the Dirac equation (6). Suppose a pair $\left(\Psi, \bar{A}_{i}\right)$ is given that solves (6), then $\bar{A}_{i}$ may be expressed in terms of the zero mode $\Psi$ as

$$
\begin{gathered}
\bar{A}_{i}=\frac{1}{|\vec{\Sigma}|}\left(\frac{1}{2} \epsilon_{i j k} \partial_{j} \Sigma_{k}+\operatorname{Im} \Psi^{\dagger} \partial_{i} \Psi\right) \\
=\frac{1}{2} \epsilon_{i j k}\left(\partial_{j} \ln |\vec{\Sigma}|\right) \mathcal{N}_{k}+\frac{1}{2} \epsilon_{i j k} \partial_{j} \mathcal{N}_{k}+\operatorname{Im} \widehat{\Psi}^{\dagger} \partial_{i} \widehat{\Psi}
\end{gathered}
$$

where we have expressed $\bar{A}_{i}$ in terms of the general unit vector and unit spinor

$$
\overrightarrow{\mathcal{N}}=\frac{\vec{\Sigma}}{|\vec{\Sigma}|}, \quad \widehat{\Psi}=\frac{\Psi}{\left|\Psi^{\dagger} \Psi\right|^{1 / 2}} .
$$

Now we introduce the zero modes

$$
\Psi^{(M)}=e^{i \Lambda} e^{M / 2} \Psi
$$

where $\Psi$ is the zero mode (9) and $M$ is an (at the moment arbitrary) function of $T$. The pure gauge factor $\Lambda$ will be determined accordingly below. Due to the fact that $T_{, i} \Sigma_{i} \equiv T_{, i} B_{i}^{(1)}=0$ (where $B_{i}^{(1)}$ is given in (11)), which is obvious from (12), it is still true that $\Sigma_{i, i}^{(M)}=0$, i.e., $\Psi^{(M)}$ really is a zero mode. The corresponding gauge field $\bar{A}_{i}^{(M)}$ that solves the Dirac equation together with $\Psi^{(M)}$ reads (we use (26))

$$
\bar{A}_{i}^{(M)}=\bar{A}_{i}^{(0)}+\frac{1}{2} \epsilon_{i j k}\left(\partial_{j} M\right) N_{k}+\Lambda_{, i}
$$


where $\bar{A}_{i}^{(0)}$ is the gauge field (10) plus the background gauge field (4), and $\vec{N}$ is the specific unit vector (5). For the corresponding magnetic field $\bar{B}_{i}^{(M)}$ we find

$$
\bar{B}_{l}^{(M)}=\bar{B}_{l}^{(0)}+\frac{1}{2}\left[M^{\prime}\left(T_{, l k} N_{k}+T_{, l} N_{k, k}-T_{, k k} N_{l}-T_{, k} N_{l, k}\right)-M^{\prime \prime}\left(T_{, k}\right)^{2} N_{l}\right]
$$

where we have used $M_{, k}=M^{\prime} T_{, k}, T_{, k} N_{k}=0$ and $\left(\vec{N}^{2}\right)_{, k}=0$. After some tedious algebra, we arrive at the expression

$$
\bar{B}_{l}^{(M)}=\bar{B}_{l}^{(0)}-\frac{8(1+T)^{2}}{\left(1+r^{2}\right)^{2}}\left(M^{\prime}+T M^{\prime \prime}\right) N_{l}
$$

Now we want to insert this into the second equation, (7) (the Chern-Simons equation). Therefore, we have to subtract the background field $(4), \bar{B}_{i}^{(0)}-B_{i}^{\mathrm{B}}=B_{i}^{(1)}=16(1+$ $\left.r^{2}\right)^{-2} N_{i}$. We find that

$$
\bar{B}_{l}^{(M)}-B_{l}^{\mathrm{B}}=\frac{16}{\left(1+r^{2}\right)^{2}} N_{l}-\frac{8(1+T)^{2}}{\left(1+r^{2}\right)^{2}}\left(M^{\prime}+T M^{\prime \prime}\right) N_{l} \stackrel{!}{=} \Sigma_{l}^{(M)}=\frac{16 e^{M}}{\left(1+r^{2}\right)^{2}} N_{l}
$$

or, after multiplication by $N_{l}$, precisely eq. (25). Hence, we have shown that, in the presence of the fixed prescribed background magnetic field (4), there exists an infinite number of fully three-dimensional solutions to the system of equations (6), (7).

Here we still should explain why $n$ is restricted to integer values, which is related to the pure gauge factor $\Lambda$ in (28). The problem is that the gauge potential (29) without the pure gauge term is singular. For the explicit expressions $M_{n},(24)$, the gauge potential (29) may be rewritten as

$$
A_{j}^{\left(M_{n}\right)}=A_{j}-\frac{(n-1)\left(1-T^{n+1}\right)+(n+1)\left(T-T^{n}\right)}{(1+T)\left(1+T^{n}\right)} \sigma_{, j}+(n-1) \Lambda_{, j}
$$

where

$$
\Lambda=\sigma^{(1)}-\sigma^{(2)}
$$

is chosen such that (33) is regular everywhere $\left(\sigma^{(1)}\right.$ and $\sigma^{(2)}$ are defined in (22)). This implies that $n$ has to be integer, because only for integer $n \exp (i(n-1) \Lambda)$ (and consequently the spinor (28)) will be single-valued. Further, we may compute the resulting Chern-Simons density

$$
\vec{A}^{\left(M_{n}\right)} \cdot \vec{B}^{\left(M_{n}\right)}=\frac{64 n^{3}}{\left(1+r^{2}\right)^{3}} \frac{T^{n-1}(1+T)^{2}}{\left(1+T^{n}\right)^{2}}
$$

which, when expressed in spherical polar coordinates $(r, \vartheta, \varphi)$ depends on $r, \vartheta$ only. By integrating (35) we can explicitly verify the relation $N=n^{2}$ for the Hopf index $N$. We have not succeeded in integrating (35) analytically so far, but (35) may easily be integrated in $r, \vartheta, \varphi$ coordinates numerically with the help of mathematica. The integrand is so well behaved that the numerical integration reproduces the integer result $N=n^{2}$ without showing even a small numerical deviation. The only technicality is that for large $n$ one has to subdivide the range of integration for $\vartheta \in[0, \pi]$, because the integrand (35) becomes rather oscillatory for large $n$. 


\section{Discussion}

We have shown that, in the presence of the fixed prescribed background magnetic field (4), there exists an infinite number of fully three-dimensional solutions to the system of equations (6), (7). Further, these solutions (i.e., the magnetic fields) are the Hopf curvatures of the Hopf maps (16), (20) and are, therefore, labelled by the corresponding Hopf index $N=n^{2}, n \in \mathbf{Z}$.

Here the background field (4) was crucial, because it determines the inhomogeneous part of the non-linear differential equation (25), and, because of this non-linearity, the inhomogeneous part of (25) crucially affects the nature of the solutions of (25). Differently stated, the Hopf instanton solutions (24) uniquely determine the background field (4).

Before closing, we want to briefly discuss whether it is possible to further interpret the background field (4), beyond just stating that its presence is crucial for the existence of the solutions (16), (20). Indeed, there are (at least) two interpretations that we want to describe now.

Firstly, let us study the following zero modes $\Psi_{l}=\exp \left(i \Lambda+M_{l} / 2\right) \Psi$, analogous to (28), where we now choose

$$
M_{l}=l \ln \frac{T}{1+T}
$$

(and $\Lambda=l \varphi=l \sigma^{(1)}$ to achieve a non-singular gauge potential for $M_{l}$ ). It follows easily that

$$
\Psi_{l}=\frac{2^{l+2} r^{l}}{\left(1+r^{2}\right)^{l}}(\mathbf{1}+i \vec{x} \vec{\sigma})\left(\begin{array}{c}
Y_{l, l}(\vartheta, \varphi) \\
0
\end{array}\right)
$$

where $Y_{l, l}$ are spherical harmonics. In other words, $\Psi_{l}$ is just a higher angular momentum zero mode (with magnetic quantum number $m=l+1 / 2$ ) that can be constructed from the simplest zero mode (9), see [25, 26]. The gauge field $\bar{A}_{j}^{(l)}$ that solves the Dirac equation (6) together with $\Psi_{l}$ may be computed easily, and its magnetic field is

$$
\bar{B}_{j}^{(l)}=\frac{12+8 l}{\left(1+r^{2}\right)^{2}} N_{j}
$$

i.e., adding one unit of angular momentum to $\Psi_{l}$ changes the corresponding magnetic field by $8\left(1+r^{2}\right)^{-2} \vec{N}$, which is precisely minus two times the background magnetic field (4). It is, therefore, tempting to conjecture that the background field (4) is somehow related to the half-integer intrinsic angular momentum (i.e., spin) of the fermion. Of course, this is just an observation at this point, because a mechanism that generates this background field is still missing.

Secondly, it is possible to re-interpret the background field $\vec{A}^{\mathrm{B}},(4)$, as a spin connection $\omega$ in the Dirac equation (6) on a conformally flat manifold with torsion. Generally, the Dirac operator with spin connection reads (see e.g. 229 for details)

$$
\mathcal{D}=\gamma^{a} E_{a}{ }^{\mu}\left(\partial_{\mu}+A_{\mu}+\frac{1}{4}\left[\gamma_{b}, \gamma_{c}\right] \omega^{b c}{ }_{\mu}\right)
$$


where $\gamma^{a}$ (三 $\sigma^{a}$ in our case) are the usual Dirac matrices, $E_{a}{ }^{\mu}$ is the inverse vielbein and $\omega^{b c}{ }_{\mu}$ is the spin connection (here $\mu, \nu$ are Einstein (i.e., space time) indices and $a, b, c$ are Lorentz indices). Our Dirac equation (6) may be rewritten in the form of eq. (39) provided that the vielbein is conformally flat, $E_{a}{ }^{\mu}=f \delta_{a}^{\mu}$, where $f$ is an arbitrary function. Using $\left[\sigma_{b}, \sigma_{c}\right]=2 i \epsilon_{b c d} \sigma^{d}$ we find

$$
\frac{i}{2} \delta_{a}^{k} \epsilon_{b c d} \sigma^{a} \sigma^{d} \omega_{k}^{b c} \stackrel{!}{=} \delta_{a}^{k} \sigma^{a} A_{k}^{\mathrm{B}}
$$

(here $k$ is an Einstein index in three dimensions). The 1.h.s. of (40) has to be antisymmmetric in $a, d$, i.e., the quantity $\widetilde{\omega}_{d a}:=\delta_{a}^{k} \epsilon_{b c d} \omega^{b c}{ }_{k}$ obeys $\widetilde{\omega}_{d a}=-\widetilde{\omega}_{a d}$. This leads to $\widetilde{\omega}_{a b}=\epsilon_{a b c} \delta_{c}^{k} A_{k}^{\mathrm{B}}$. If we further assume $\omega^{a b}{ }_{k}=-\omega^{b a}{ }_{k}$ (i.e., covariant constancy of the metric) then we find that

$$
\omega_{a b k}=\delta_{k a} A_{b}^{\mathrm{B}}-\delta_{k b} A_{a}^{\mathrm{B}}
$$

(where $A_{a}^{\mathrm{B}} \equiv \delta_{a}^{k} A_{k}^{\mathrm{B}}$, i.e., it is not the Lorentz vector $E_{a}{ }^{k} A_{k}^{\mathrm{B}}$ ). Finally, we find for the torsion $T$ (expressed in Lorentz indices only)

$$
2 T_{a b c}=\left(\delta_{a b} \delta_{c}^{k}-\delta_{a c} \delta_{b}^{k}\right) \partial_{k} f-\left(\omega_{a b c}-\omega_{a c b}\right)
$$

where

$$
\omega_{a b c}=E_{c}{ }^{k} \omega_{a b k}=f \delta_{c}^{k} \omega_{a b k} .
$$

Hence, with $\omega_{a b k}$ given by (41), we may freely choose a conformally flat metric (i.e., conformal factor $f$ ) and compute the resulting torsion via (42). Due to the form of $\omega_{a b k}$ (i.e., $\overrightarrow{A^{\mathrm{B}}}$ ) it is, however, not possible to choose a conformal factor such that the torsion is zero. On the other hand, it is possible to choose the flat metric $f=1$, so that (the anti-symmetric part of) the spin connection is given just by the torsion.

\section{Acknowledgments}

The authors thank M. Fry for helpful discussions. In addition, CA gratefully acknowledges useful conversations with R. Jackiw. CA is supported by a Forbairt Basic Research Grant. BM gratefully acknowledges financial support from the Training and Mobility of Researchers scheme (TMR no. ERBFMBICT983476).

\section{References}

[1] E. A. Kuznetsov and A. V. Mikhailov, Phys. Lett. A77 (1980) 37

[2] M. Giovannini, hep-ph/9906241

[3] R. Jackiw and S.-Y. Pi, hep-th/9911072

[4] A. M. Kosevich, B. A. Ivanov and A. S. Kovalev, Phys. Rep. 194 (1990) 117

[5] A. F. Ranada, J. Phys. A25 (1992) 1621 
[6] A. F. Ranada and J. L. Trueba, Phys. Lett. A202 (1995) 337

[7] L. Faddeev and A. Niemi, Nature 387 (1997) 58

[8] L. Faddeev and A. Niemi, hep-th/9705176

[9] J. Gladikowski and M. Hellmund, Phys. Rev. D56 (1997) 5194

[10] R. Battye and P. Sutcliffe, hep-th/9811077

[11] R. Battye and P. Sutcliffe, Phys. Rev. Lett. 81 (1998) 4798

[12] H. Aratyn, L. A. Ferreira and A. H. Zimerman, Phys. Rev. Lett. 83 (1999) 1723

[13] S. Deser, R. Jackiw and S. Templeton, Phys. Rev. Lett. 48 (1982) 975

[14] S. Deser, R. Jackiw and S. Templeton, Ann. Phys. 140 (1982) 372

[15] R. Jackiw, K. Lee and E. J. Weinberg, Phys. Rev. D42 (1990) 3488

[16] R. Wang, Comm. Math. Phys. 137 (1991) 587

[17] R. Jackiw and S.-Y. Pi, Prog. Theor. Phys. (Suppl.) 107 (1992) 1

[18] G. Dunne, hep-th/9902115

[19] S. C. Zhang, T. H. Hansson and S. Kivelson, Phys. Rev. Lett. 62 (1989) 82

[20] J. K. Jain, Phys. Rev. Lett. 63 (1989) 199

[21] A. N. Redlich, Phys. Rev. D29 (1984) 2366

[22] O. Alvarez, Comm. Math. Phys. 100 (1985) 279

[23] A. Polychronakos, Nucl. Phys. B281 (1987) 241

[24] R. Dijkgraaf and E. Witten, Comm. Math. Phys. 129 (1990) 393

[25] M. Loss and H.-Z. Yau, Comm. Math. Phys. 104 (1986) 283

[26] C. Adam, B. Muratori and C. Nash, Phys. Rev. D60 (1999) 125001

[27] C. Houghton, N. Manton and P. Sutcliffe, Nucl. Phys. B510 (1998) 507

[28] P. A. Horvathy, hep-th/9903116

[29] R. A. Bertlmann, "Anomalies in Quantum Field Theory", Clarendon Press, Oxford 1996 